\title{
Color: what, how, and why we see: a workshop for $\mathrm{K}-12$ students and parents
}

Katherine Calabro, G. Groot Gregory, Michael Zollers

Katherine W. Calabro, G. Groot Gregory, Michael W. Zollers, "Color: what, how, and why we see: a workshop for K-12 students and parents," Proc. SPIE 9188, Optics Education and Outreach III, 91880K (15 September 2014); doi: 10.1117/12.2060856

Event: SPIE Optical Engineering + Applications, 2014, San Diego, California, United States 


\title{
Color: What, How and Why We See - A Workshop for K-12 Students and Parents \\ Katherine Calabro, G. Groot Gregory, and Michael W. Zollers \\ Synopsys, Inc., 377 Simarano Dr., Marlborough, MA 01752
}

\begin{abstract}
Through the ongoing educational outreach activities of the NES/OSA, we have been invited on several occasions to present optics workshops to students of many ages and backgrounds. With a nearly-overwhelming plethora of optics topics that could be presented, we have decided to develop a curriculum on color science that can be presented in a workshop format. Color science was chosen due to the wealth of examples of the application of color within a student's culture, society, technology, and experiences.

The goal of the workshop is to teach basic color science by examining objects and events that the students can experience or interact with in their own lives. The curriculum can be scaled to match groups of different sizes and backgrounds as well as to fit within certain time constraints. Depending on logistics, a variety of hands-on demos can be presented, or the workshop can be fully tutorial-based. This curriculum has been presented several times and is constantly evolving based upon each experience.

In this paper, we present the portions of the curriculum that have been developed to date. We discuss considerations for adding or removing sections to meet specific workshop constraints. We will also present the evolution of the curriculum from inception to its current state, highlighting the lessons learned from each presentation of the curriculum.
\end{abstract}

Keywords: Color Science, Education, Outreach, Workshop, Curriculum

\section{INTRODUCTION AND MOTIVATION}

The New England Section of the Optical Society of America (NES/OSA) has been using optics demonstrations for education outreach, most recently at local science fairs, since the mid-1970s [1]. Every year we participate in more events, usually at the invitation of those who have visited us in years past as they create and expand their own events. Some of the local science fairs and STEM events have moved beyond what might be called an "exhibition" format and started adding focused workshops that can be attended by students and parents alike.

Toward the end of 2013, we were invited to participate in our usual assortment of events during the spring of 2014 and, surprisingly, two separate event organizers inquired about us leading a workshop of our choosing. Despite not having a topic, much less the materials to present, we accepted both invitations and promised to provide details about the topic (once they became available).

In the end, we chose to develop a curriculum around topics in color science. A lot of factors played into this decision. Some of these factors include, but are not limited to:

- A wealth of topics, allowing us to pick and choose as time allows,

- A general lack of understanding of the science among the audience,

- And the realization that audience members should have each experienced many aspects of color, whether they know it or not.

In this paper, we review the primary and secondary goals of the curriculum, present the curriculum, and discuss our experiences gained during its presentation at two events in during the spring of 2014.

\section{GOALS}

As with any curriculum, the primary goal is to educate. In this case, our aim is to impart knowledge about various aspects of color science to the members of the audience. Given the events at which we were invited to participate, the audience for these materials typically includes children and young adults (as well as their parents) who have an interest in STEM (Science, Technology, Engineering, and Math). In addition to answering the audience's current

Optics Education and Outreach III, edited by G. Groot Gregory, Proc. of SPIE Vol. 9188,

91880K () 2014 SPIE · CCC code: 0277-786X/14/\$18 · doi: 10.1117/12.2060856 
questions about color, we also want to incite curiosity; it's impossible to cover everything, so we endeavor to provide the basics and make the topic interesting enough for the students to want to do their own follow-on inquiry.

A secondary goal was to develop a set of materials that allow us to tailor the presentation to a variety of scenarios, fitting the curriculum to the time allotted and audience demographics. Even with a small sample size of three workshops (we presented twice at one event), it is apparent that the amount of time we will be given to present will vary greatly: one event gave us 75 minutes per session, the other 45 minutes. Also, one event hosted only girls without accompanying adults while the other was mixed gender and included adults.

\section{THE CURRICULM}

With these goals in mind, we have developed a modular color science curriculum with materials suitable for a variety of audience demographics and logistics. To achieve this, we identified three over-arching themes that we reinforce with as much material as time allows. These themes are:

- What is Color?

- What are the Sources of Color?

- How is Color Used?

If possible, a fourth theme, centered on "other" types of color can be presented. Specific topics and demonstrations that can be used for each the themes are presented in the following sections.

\subsection{What is Color?}

We start each session by asking a very simple, open-ended question to the audience members: "What is Color?" Our hope is to establish early that we are looking for feedback and interaction, and also to generate ideas that we can reinforce during the remaining time for the course. Color means a lot of things to different people and it is very experiential and cultural. We provide a definition for color, provided by Crayola [2], which states: "Color is the aspect of things that is caused by differing qualities of light being reflected or emitted by them."

Further materials presented for this section speak to answering the question posed. The presenters can go as deeply into the rabbit hole as they desire for their audience. Our goal is to establish a common vocabulary and to explain and demonstrate how the terms describe the colors and mechanisms for color that we see around us. For this section, we defined three categories of material to present. The three categories are:

- The Nature of Light

- How We See

- Making Colors

\subsubsection{The Nature of Light}

Color, as we know it, is a reaction of the human visual system to a light stimulus. As such, we can't describe color without first describing light. In broad terms, we describe light as a wave, and, depending on the wavelength of the light, our eyes perceive different wavelengths of light as different colors. For this section we primarily use a series of images depicting the electro-magnetic spectrum. One chart, shown in Figure 1, compares the wavelength of light to various other physical objects that have a similar size, to within an order of magnitude. 


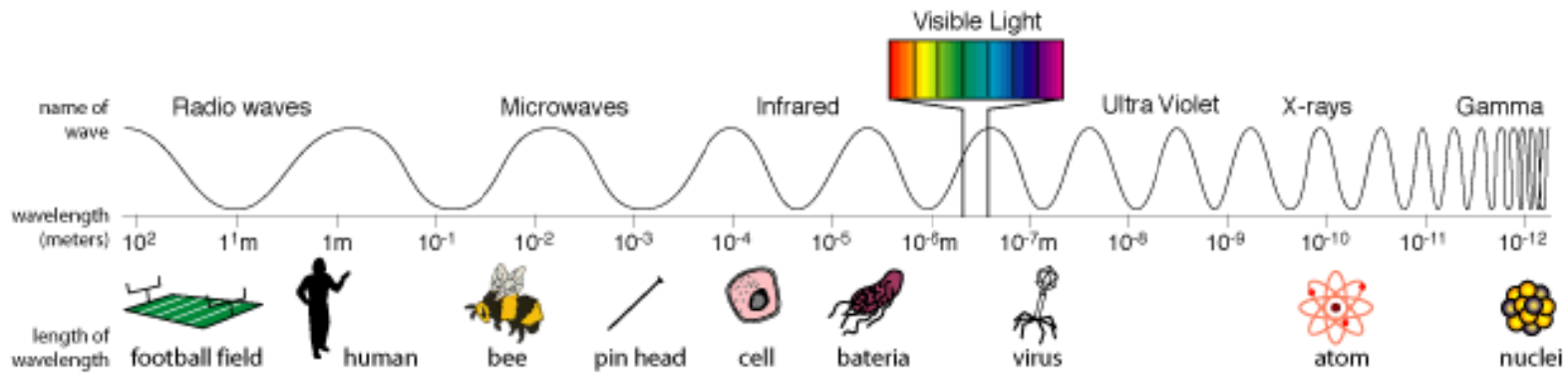

Figure 1. A depiction of the electromagnetic spectrum that shows objects whose size is similar to the wavelength of light [3].

Key Terms:

- Light

- Wavelength

- Spectrum

Materials:

- Charts and graphics depicting the electromagnetic spectrum and the relative sizes of the wavelength of light as compared to other similarly-sized objects.

\subsubsection{How We See}

Once the audience understands the role of light in generating colors, they need to understand the role of our eyes and brain, the two principle components of the human visual system. The cones in our retina produce chemicals that are converted into electrical signals at the optic nerve. Our brain interprets the electrical signals and forms an image in our mind. Different amounts of chemicals produced by the three types of cones allow our brain to interpret a wide color gamut using this tristimulus system. The presentation can delve further into the role of the brain in determining color, time and interest permitting.

To explain the workings of the visual system, we show a series of graphics depicting how light is focused through the eye onto the retina, shown in Figure 2, and describe how the different responsivities of the three types of cones create a tristimulus system that processes the visible portion of the electromagnetic spectrum. A close-up of the visible spectrum is shown, such as in Figure 3, and we ask the question: "What color is missing?" The answer is invariably: "white".

\section{Photoreceptors}

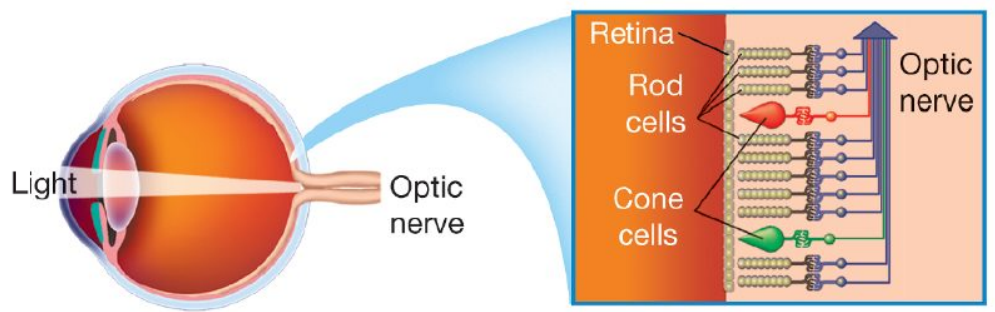

Figure 2. Diagram of a human eye showing light entering through the lens and focusing onto the retina.[4] 


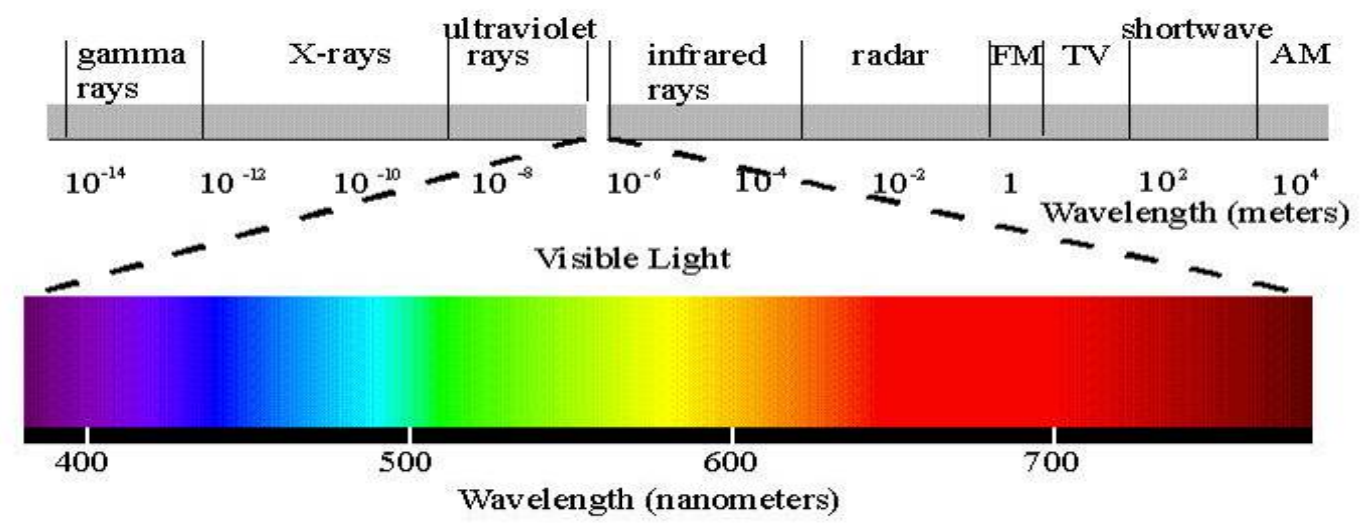

Figure 3. Visible portion of the electromagnetic spectrum showing the typical ROYGBIV (inverted) progression. [5]

White, as with most of the colors we perceive, is a combination of different wavelengths of visible light. To explain this, we show a CIE 1931 Chromaticity diagram, as depicted in Figure 4. This diagram represents the color gamut possible for the average human observer. Colors composed of a single wavelength of light are represented on the outer hull of the diagram, and the remaining colors within are combinations of multiple wavelengths.

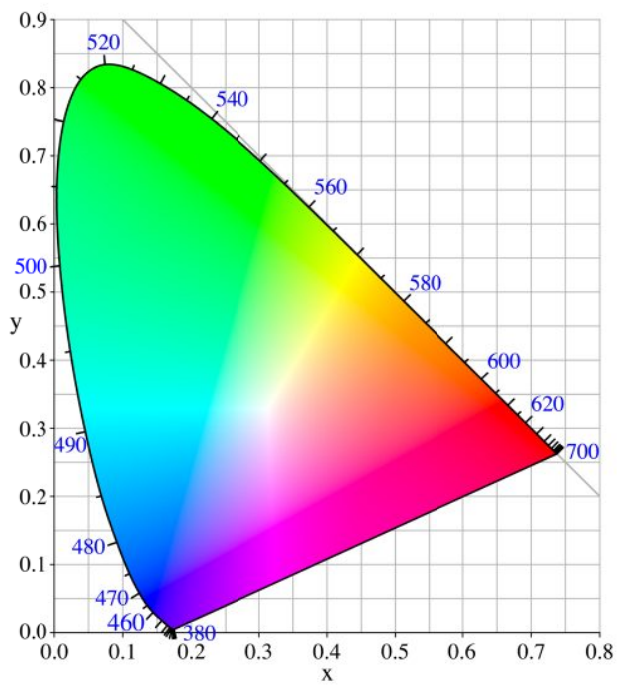

Figure 4. 1931 CIE Chromaticity Diagram depicting the visible color gamut of the average human observer. [6]

\section{Key Terms:}

- Retina

- $\quad$ Rods and Cones

- Tristimulus

- Chromaticity

- Color Gamut

Demonstrations:

- This is a good time to bring up images showing how easy it is to trick your brain with visual stimulus. There is an endless supply of "optical illusions" that can be found to demonstrate such concepts as simultaneous contrast, eye jitter, etc. 


\subsubsection{Making Colors}

This section covers the mechanisms that create colors, specifically, the combinations of wavelengths of visible light, or spectra. Primarily, colors can be added or subtracted to create new colors. The colors can overlap spatially, or they can be temporally combined by the brain. The spatial overlap can occur through superposition or by tiling at sizes smaller than the resolvable limit of the eye. Each of these processes can be placed in the context of common applications like displays and printing.

Most attendees are familiar with subtractive color. Printing and painting use a subtractive process. Additive color works with light sources and is a less common topic; however, most display devices that the attendees experience in their daily lives use an additive process. It's important to talk about both processes and show visuals, such as Venn diagrams of the overlapping primaries as shown in Figure 5.

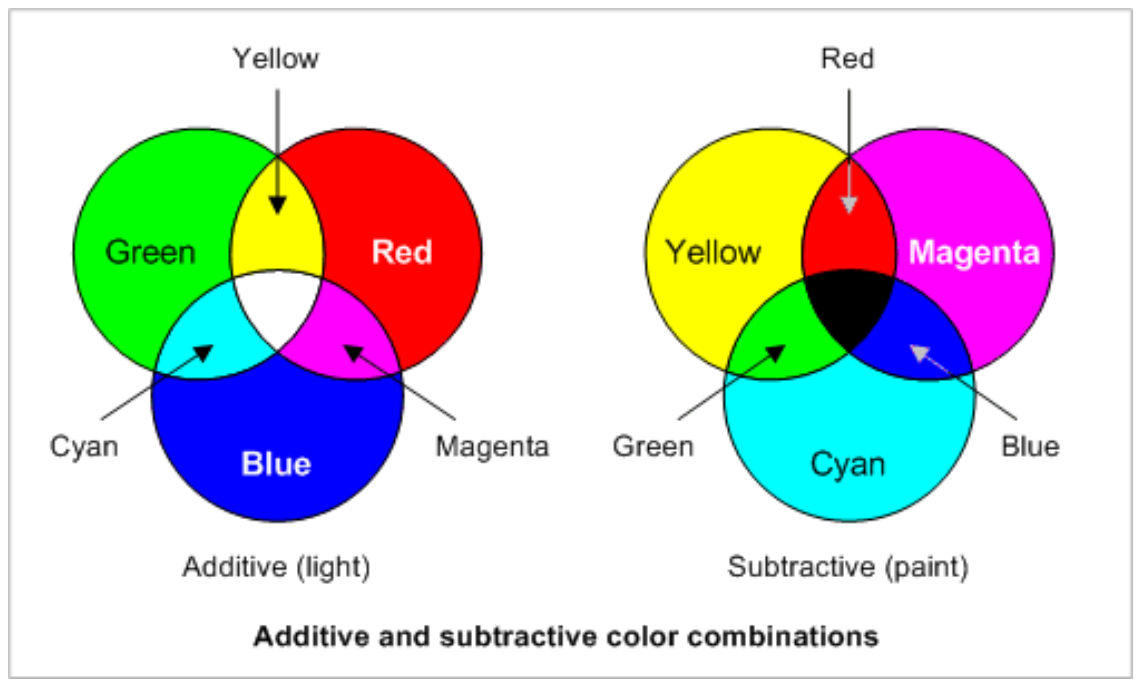

Figure 5. Venn diagrams showing how the combinations of different additive or subtractive primaries interact. [7]

Combining the concepts of chromaticity, color gamut, and additive color, we can introduce color rendering and metamerism. Using just a two-stimulus color gamut, we can show that there are a near-infinite number of combinations of primaries that can create any color within the chromaticity chart. This is an example of metamerism and leads to a discussion of why certain combinations of wavelengths are more favorable than others, which is an example of why color rendering is important.

Color rendering is a fairly simple concept to describe. We show images of objects under various illumination conditions and show how the spectral content of the illuminant changes the appearance of the object. Figure 6 shows how certain colored fruits appear under different illuminants. We also show a textile example for colored clothing. Figure 7 shows a red shirt and blue pants under white light, then red light, and finally blue light. Since the clothes selectively reflect certain colors, if the illuminant is deficient, the cloth appears black. 
Good light quality

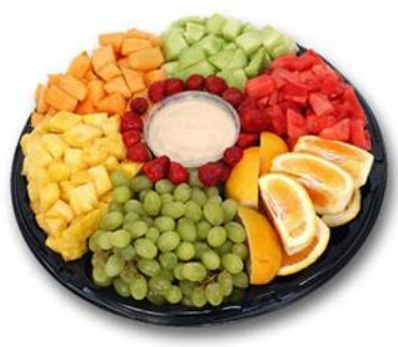

LED Lamps

CRI 80 or above
Bad light quality

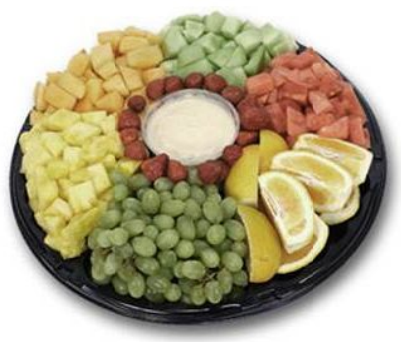

Fluorescent Lamps CRI 70 or below

Figure 6. A fruit platter under two different lighting conditions, one with good color rendering and one without. [8]
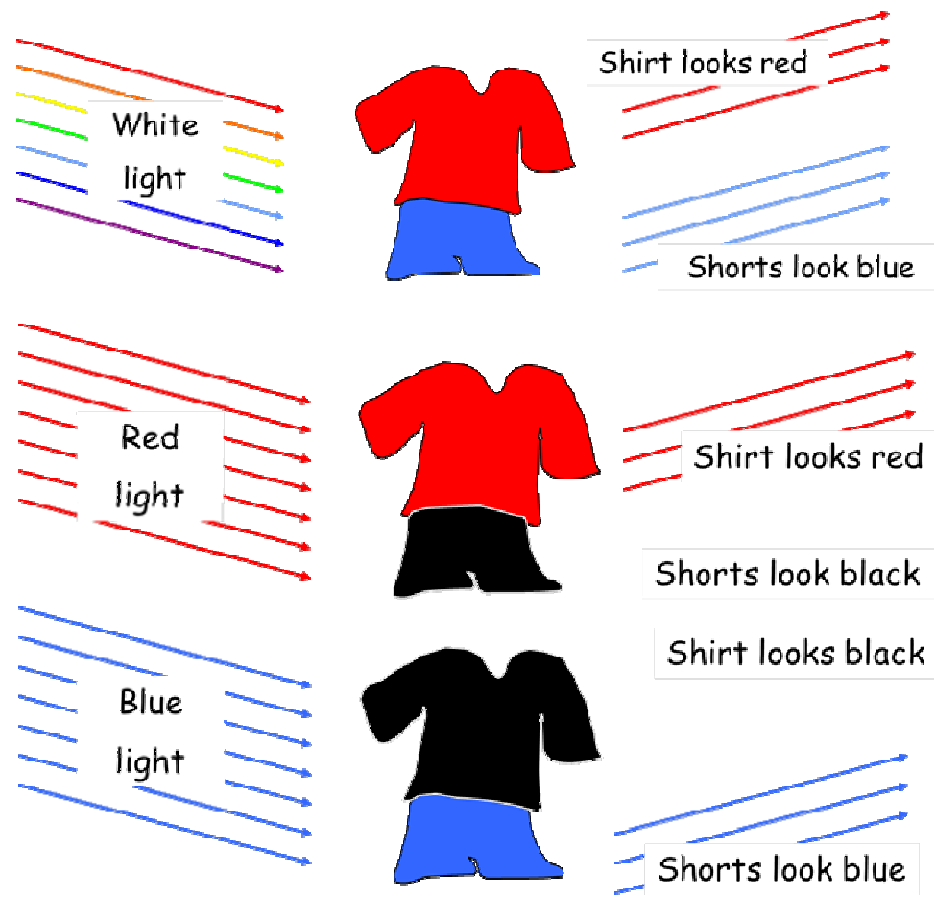

Shirt looks black

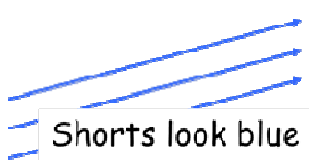

Figure 7. Color rendering of clothing under various lighting conditions. [9]

We have described how colors are typically composed of many other, single-wavelength colors. To visualize this, we hand out transmissive diffraction gratings. We then ask the audience to observe a variety of white light sources through the gratings. The grating separates the source's light by wavelength and angle, so the attendees get to see the component colors of the source. The grating is a useful tool later in the curriculum and makes a nice gift to take home. However, it is very important to remind the attendees that they should not look into the sun through the grating. Figure 8 shows what can be seen through the diffraction grating. 


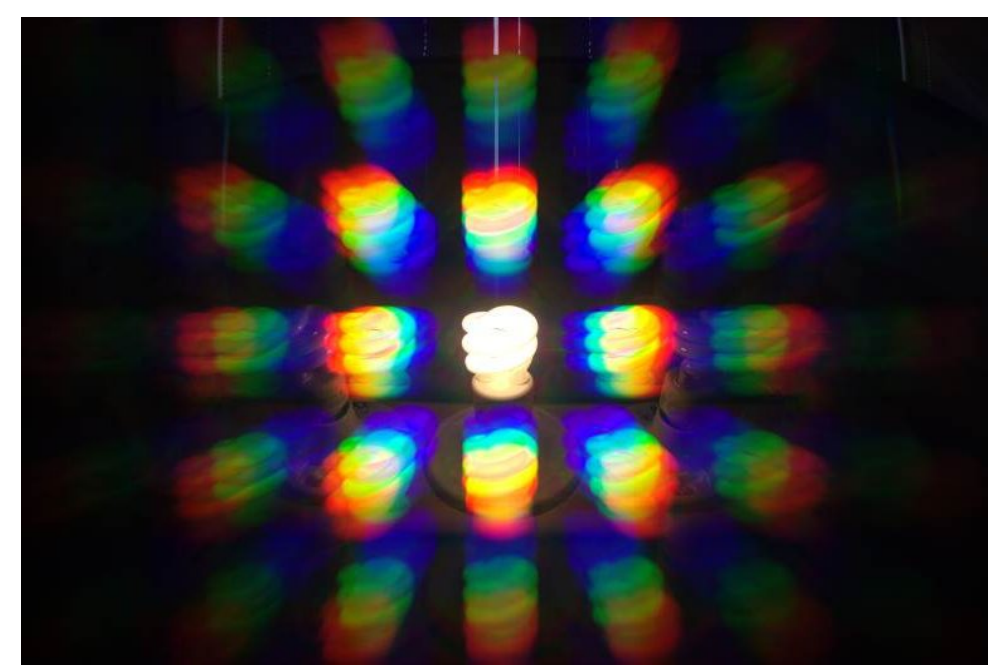

Figure 8. A 3500K compact fluorescent lamp as viewed through a transmissive diffraction grating.

Lastly, we discuss dispersion as being another method to separate light into its component wavelengths. A picture of a dispersive prism, shown in Figure 9, is provided. We also describe dispersion as the mechanism that produces rainbows, with supporting imagery, as depicted in Figure 10.

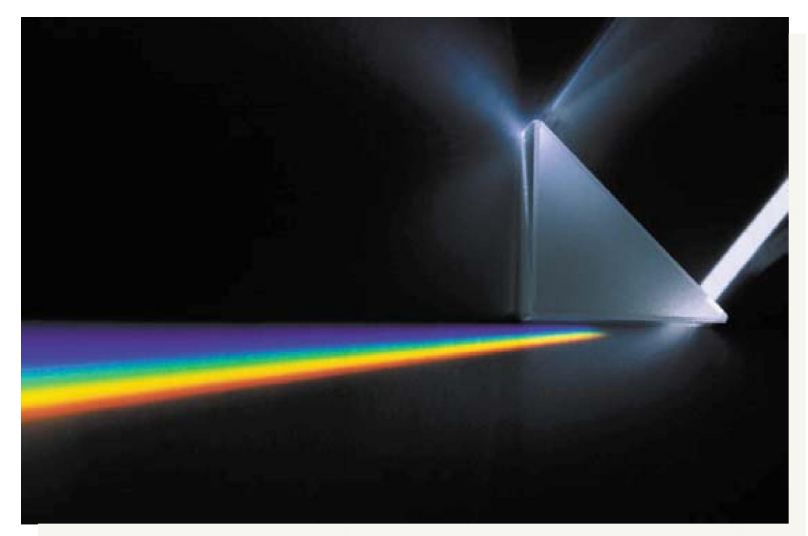

Figure 9. An image showing the effect of dispersion on a beam of white light as it passes through a right-angle prism. [10] 

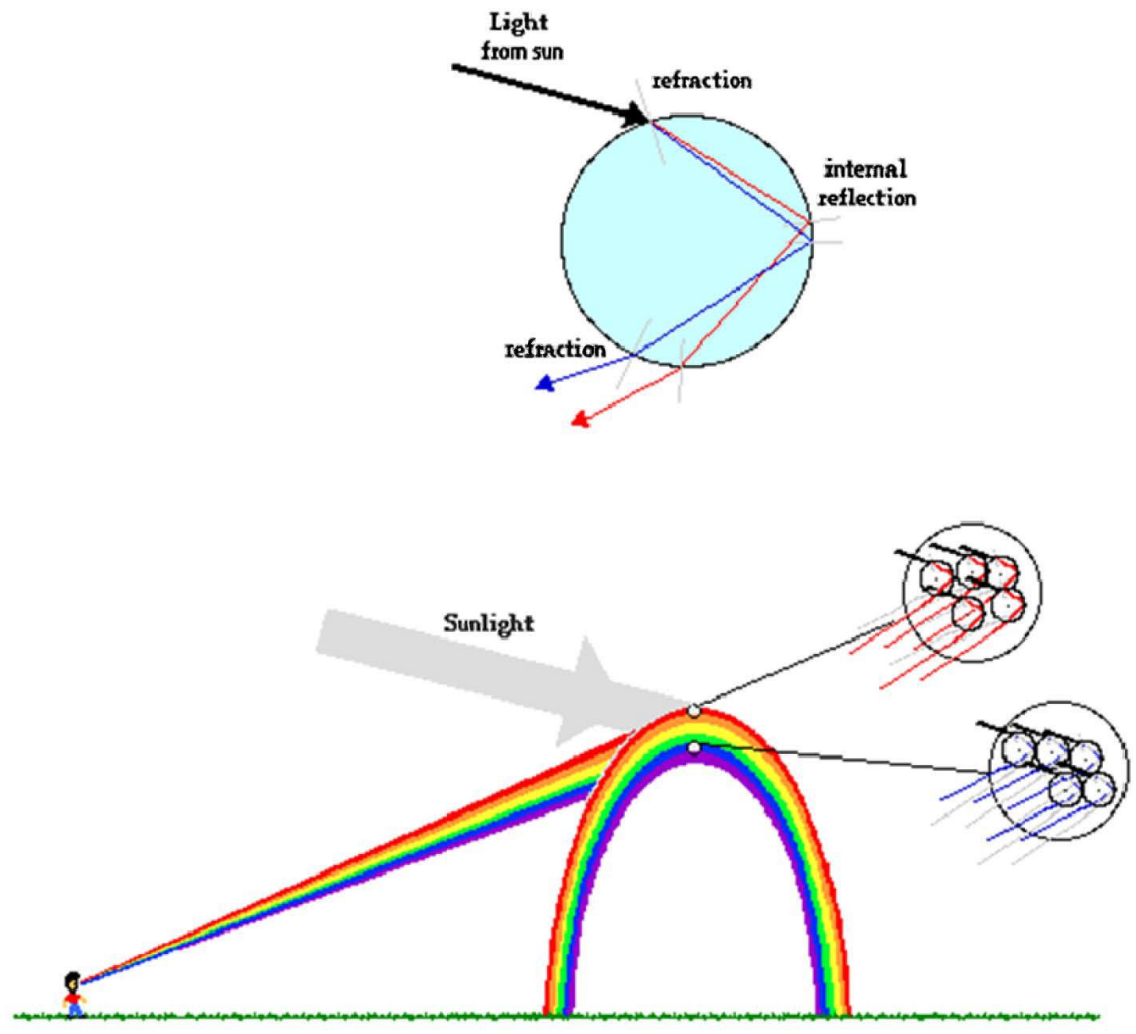

Figure 10. Graphical depiction of how rainbows are formed by the dispersion of light through water droplets. [11]

Key Terms:

- Additive Color

- Subtractive Color

- Dispersion

- Color Rendering

- Metamerism

- Spatial Addition or Subtraction

- Temporal Addition

\section{Demonstrations:}

- For additive color, shine a red, green, and blue light source onto the wall and demonstrate how the colors add to create new colors.

- An interactive, hands-on demo that uses coffee filters and water to separate the colored dyes used in markers illustrates subtractive color [12]. This demo takes time.

- If absorptive theatrical filters, or gels, are available, overlapping different gels in front of an overhead projector demonstrates how the first filter absorbs certain wavelengths and modifies the effect of subsequent filters in the stack.

- If transmissive filters are available, they can be used to show that the light that isn't transmitted is reflected, so the filter appears a different color than the transmitted light. 
- Transmissive diffraction gratings. Allow the audience to view a variety of light sources through the diffraction grating. What they see are images of the source, separated by wavelength as a function of angle. By viewing different sources, especially white sources, they can see that different "colors" of light are typically not pure, but are instead composed of many other colors.

\subsection{What are the Sources of Color?}

To answer this question, we show the audience various light sources, explain how they generate light, and why their spectrum is different from other light sources. The first type of source discussed is incandescent. These include our sun, candles, and conventional light bulbs (although that reference is becoming obsolete). For these sources, the color spectrum is determined by the source's temperature. This leads to another example of an incandescent source with which many of them are familiar: the heating element from an electric stove.

Next, we describe several types of luminescence, starting with bioluminescence. There are many examples of bioluminescence that are familiar to the audience and several that are common in pop culture, which we also highlight. Fireflies are the primary example in nature, and Hollywood has provided several examples of bioluminescence in recent films that many young attendees have seen. These movies include Finding Nemo and The Life of Pi.

Fluorescence is one type of photoluminescence. Fluorophores are used in many commercial lighting devices, such as compact fluorescent bulbs and fluorescent tubes. To demonstrate photoluminescence and fluorescence, we have several examples of phosphors and fluorophores that excite under blue or ultra-violet light. Using blue and UV laser diodes, we can excite the fluorophores and show how they absorb the energy of the photons, hold on to the energy for a varying amount of time, then release the energy, usually in the form of light with a longer wavelength. Offering laser pointers to volunteers to draw on a phosphor sheet, where one participant is given a green laser and the others receiving blue lasers, provides a fun learning exercise.

We conclude the section on light sources with Electro-luminescence. The same energy transitions happen with these materials as they do with fluorophores; however, the excitation energy comes from an electrical source, not a photon. We have several examples of electro-luminescent (EL) wire and panels of different colors that we show to the group; these have recently become easier to obtain at hobby and electronics stores. Additionally, electroluminescence is the mechanism that allows light-emitting diodes (LEDs) and solid-state lasers to generate light. We have many examples of LED and laser pointers that demonstrate these principles.

\section{Key Terms:}

- Lasers

- LEDs

- Incandescence

- Photoluminescence

- Electro-luminescence

- Bioluminescence

- Fluorescence

- Phosphorescence

Demonstrations:

- Procure and demonstrate the different types of sources.

- Laser - use a laser pointer (different wavelengths help)

○ LED - an example of electro-luminescence. Easy to obtain.

○ EL Wire or Panels - an example of electro-luminescence. Getting easier to obtain.

Incandescence - Light bulb (automotive if household is no longer available) 
- Photoluminescence - Obtain glow in the dark safety tape and excite it using a blue light source (LED or laser)

\subsection{How is Color Used?}

We answer this question by placing the color science previously described into the context of various applications demonstrating its use. It is a "how it's made" for a variety of devices that the audience experience in their everyday life. Generally, we pull in and describe examples from the following areas:

- Displays (additive and subtractive)

- Printing/Art (subtractive)

- Machine Vision (subtractive)

- Medical Imaging (bioluminescence)

- Photography (subtractive)

- Textiles (subtractive)

Before describing the actual devices, it's useful to augment the optics education of the audience by describing the different ways in which these devices manipulate light. These topics include reflection, refraction, absorption, and transmission. Reflection and refraction primarily describe how light moves around the devices while absorption, transmission, and reflection are all opportunities to change the color of the light.

Color displays surround us in our everyday life, from televisions to projectors to cellphone screens. There are several ways to make displays. The most prevalent displays, cellphones and LCD televisions or monitors, start with a white or colored source, use transmission filters to subtract all but red, green, or blue light into sub-pixels, then spatially combine the combination of red, green, and blue sub-pixels to form one colored pixel via tiling. An example of this is shown in Figure 11. Projectors operate on a similar principle, but can spatially (typically via superposition) or temporally (via a spinning color wheel) combine the red, green, and blue pixels to form the image. Figure 12 shows the optical path through a spatially-combining projector system.

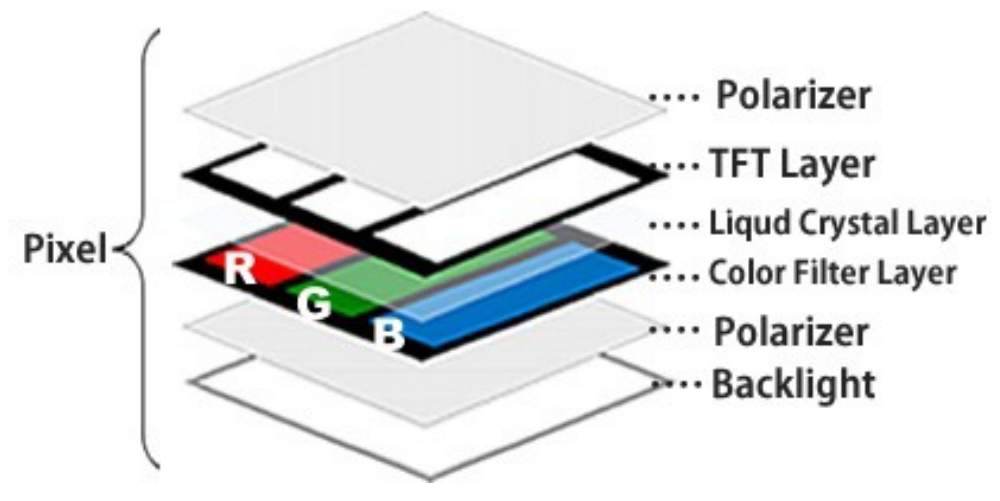

Figure 11. Depiction of the filtering and subsequent recombination of light within an LCD panel to create an image.

[13] 


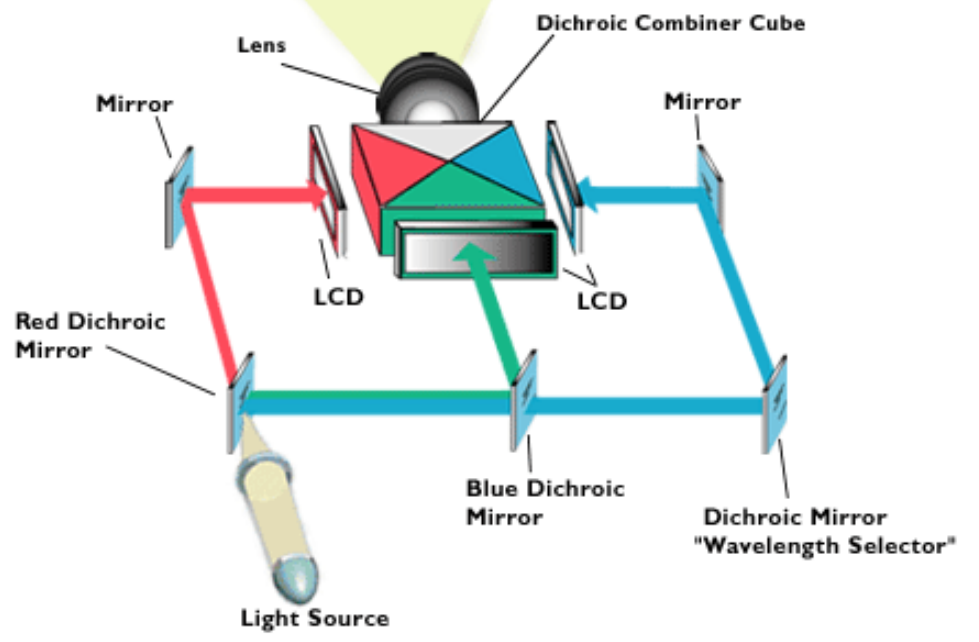

Figure 12. A diagram of the filtering and recombination of light within a projector system. [14]

Finally, we describe the typical four-color printing processes used by inkjet printers. By spatially combing cyan, magenta, yellow, and black (CMYK) dots on a sheet of paper, a wide color gamut can be produced. Diagrams that show how these dot patterns overlap are shown in Figure 13.

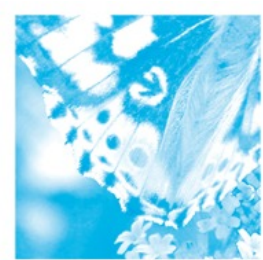

CYAN

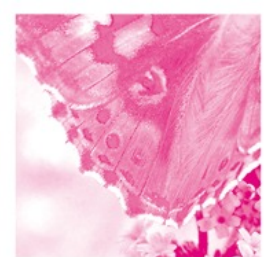

MAGENTA

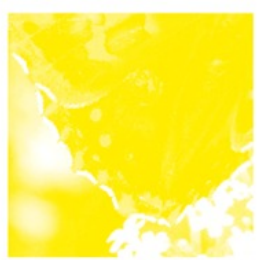

YELLOW

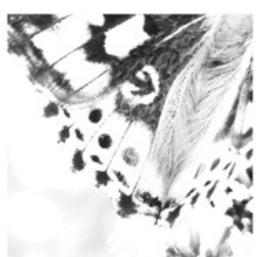

BLACK

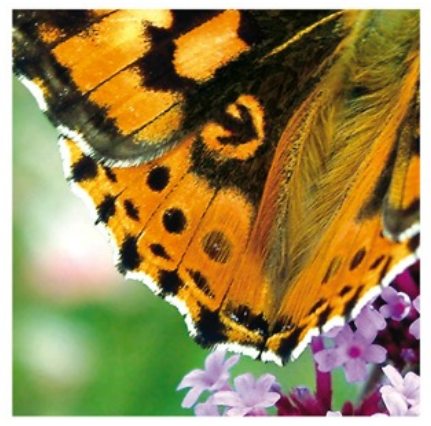

FINAL CMYK

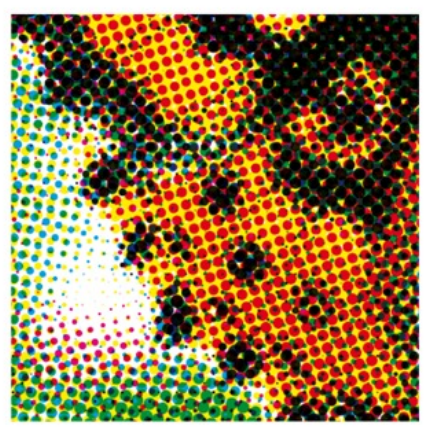

DETAIL VIEW

Figure 13. The typical CYMK printing process and the resulting dot patterns. [15]

Key Terms:

- Reflection

- Refraction

- Absorption

- Transmission 


\subsection{Other Types of Color}

This optional section is actually quite fun. In it, we talk about a variety of color topics as they apply to non-human (or abnormal human) visual systems as well as uses for the remainder of the electromagnetic spectrum. We begin by introducing the concept of alien vision, although we really mean non-human vision. It is true that we don't all see the same colors. A quick red-green color-blindness test is administered, as shown in Figure 14. This leads to a discussion of what happens in humans if not all three types of photoreceptors are working. We also describe how other animals "see" using different types of photoreceptors. An image that represents how a red-green color-blind person, or a dog (for example) see the world is shown in Figure 15. Also, a table showing the photo-sensitivities of various types of animals is presented.

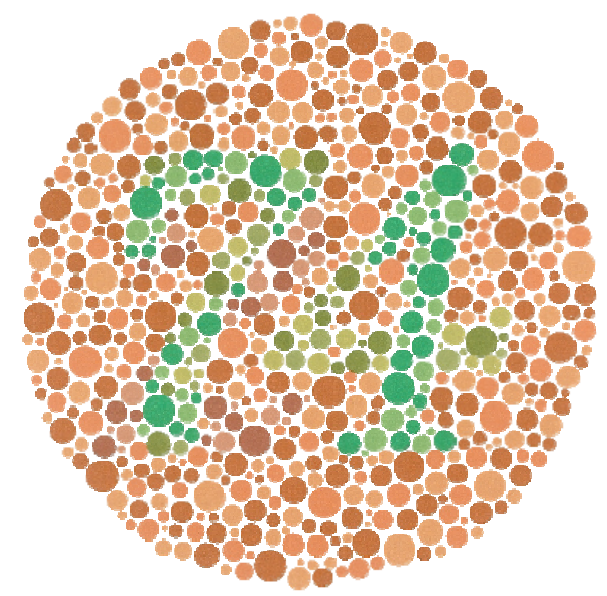

Figure 14. A simple color-blindness test. [16]

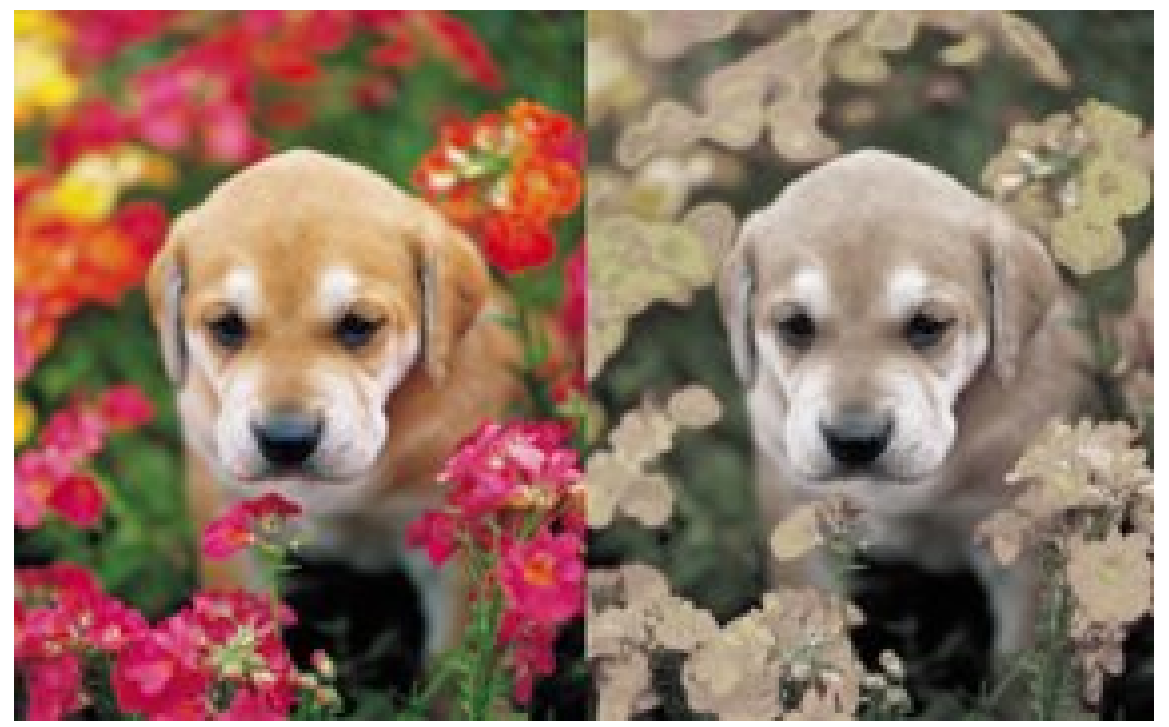

Figure 15. The image on the left shows what would be seen by a person or animal with red, green, and blue photoreceptors. The image on the right shows how a person with only two working photoreceptors perceives the same scene. [17]

Finally, we showcase uses for three other portions of the electromagnetic spectrum. We start with ultraviolet light and highlight some of its uses, from detection of material deformities to its use in locating of certain types of chemicals. We also typically have a black-lamp with us, which can be used to show the UV inks present in many driver's licenses or passports. Next, we highlight the uses of infrared light, especially as a method for detecting temperature with applications such as cameras for fire fighter to find hot spots and humans within a smoke-filled 
room or building. Lastly, we talk about the uses of x-rays familiar to most audience members, both medically and for detecting objects within other objects, such as in an airport scanner.

\author{
Key Terms: \\ - Alien vision \\ - Color blindness \\ - Infrared Imaging \\ - X-Ray Imaging \\ - Ultraviolet Imaging
}

\title{
4 GIRL SCOUT EVENT
}

We first presented this curriculum on 9 March 2014 at an event entitled, "Changing the World through STEM: Teen Career Expo", that was organized by the Girl Scouts of Eastern Massachusetts. The program included workshops, lunch with a key note speaker, and a STEM Expo of various local organizations. We had participated in a previous Expo with hands-on optics demonstrations but used the notion of a workshop to expand what NES/OSA had to offer.

Two 75 minute color workshop sessions were offered during the morning. The first session was for Cadette Level Girl Scouts, grades 6-9, and the second included Senior and Ambassador Level, grades $10-12$. Both sessions had about 20 girls participating. Two presenters conducted the workshop that was comprised of a presentation, demonstrations, and various hands-on activities. After a brief introduction of the presenters, the session started asking "What is Color?"

\subsection{Lessons Learned}

The most important lesson is that the presenters need to be prepared, both in the materials and the presentation method. Neither presenter is a teacher so there was little experience engaging students in a workshop / classroom environment. In this case, the second workshop presentation was an improvement over the first. These workshops did not have time for any pre/post testing, so no formal learning outcomes were recorded; however, the workshop offered several opportunities for the students to ask and answer questions, which were well utilized by the attendees. As a result, we judged that the topic was interesting to the students, especially during demonstrations and hand-on activities, and when the science was placed into the context of everyday life.

\section{WELLESLEY STEM EVENT}

The next workshop presentation was during the Wellesley Education Foundation's "Wellesley Science \& Technology Expo" held on 5 April 2014. The Expo was open to the public with hands-on STEM demonstrations in an exhibition layout with concurrent workshops followed by a key-note talk. The event concluded with a "Meet The STEM Professionals Student Networking Reception".

The 45 min workshops were conducted in classrooms of the Wellesley, MA high school. Our session attracted about 15 students and parents. We repurposed the materials presented at the Girl Scout event, but in order to meet the time constraints, we reduced the presentations materials. This allowed us to keep most, if not all, of the demonstrations and hands-on activities.

\subsection{Lessons Learned}

The workshop organizers had preregistered attendees for the workshops, but the actual attendance was less, which was a bit disappointing. The shortened time hurried the interactive portions of the workshop, which would have been a problem with a larger audience, so time to distribute materials needs to be taken into account.

Over the years we have learned that outreach to parents is nearly as important as it is to students; both groups can learn about the science and technology, and we believe that tech savvy parents can help encourage future STEM professionals. Hearing parents ask questions similar to those we get from the student-only groups strengthened our observations that parents can learn STEM too. It's particularly gratifying when a parent says 'That's cool'. 
A final lesson, applicable to both events, is making sure an extra set of hands attends the workshops to take photos to document the events. In the case of NES/OSA, we are able to contribute more to outreach activities by engaging more of our members. We can entice more members to become involved, and participate at more events, if we better promote the organization's activities internally.

\section{CONCLUSIONS}

Earlier this year we were provided a couple of opportunities to present optics workshops at various regional STEM events. In response, we created a curriculum of color science topics and activities that highlight both the science and applications of color, suitable for participants from a potentially wide demographic. The curriculum is modular to allow it to be tailored for a variety of lengths and tries to be comprehensive without going into too much detail about any given topic.

The curriculum was presented three times, at two different events, in the spring of 2014. The feedback, based upon the level of engagement as well as types and quality of questions asked by the audience, was generally positive. We believe that we were successful at providing a reasonable amount of information and hopefully sparked some curiosity along the way.

\section{REFERENCES}

[1] Gregory, G. G., Biss, D. P., and Darnell, B., "Optical demonstrations through science fairs," Proc. SPIE 7783, Optics Education and Outreach, 77830K (2010).

[2] “Color - What is Color?," Crayola, http://www.crayola.com/for-educators/resources-landing/articles/colorwhat-is-color.aspx (12 August 2014).

[3] "The Electromagnetic Spectrum: What is it?," http://science.hq.nasa.gov/kids/imagers/ems/ (12 August 2014).

[4] "Foundations of Physics, $2^{\text {nd }}$ Edition - Chapter 16: Light and Color (Presentation Slides)," CPO Science, http://www.cposcience.com/home/Portals/2/Media/post_sale_content/PHY2/PresentationSlides/PHY2Chp16.pp t (13 August 2014).

[5] "Physics 1230: Light and Color, Set 3 Lecture Notes: Fundamentals of Electromagnetic waves," University of Colorado at Boulder, Spring 2014, http://www.colorado.edu/physics/phys1230/phys1230_sp14/lectureNotes1230S14/lecture3_light.pptx.pdf (13 August 2014).

[6] “CIE1931xy

blank,"

Wikipedia, http://en.wikipedia.org/wiki/CIE_1931\#mediaviewer/File:CIE1931xy_blank.svg (13 August 2014).

[7] "Color Vision: One of Nature's Wonders," DIY Calculator, http://www.clivemaxfield.com/diycalculator/imgs/console-09.gif (13 August 2014).

[8] "Color Rendering Index," 2Bora, http://2bora.com/en/technologie/wspolczynnik-odwzorowania-kolorow (13 August 2014).

[9] "Light \& Color," California Subject Examinations for Teachers, California State University Northridge, http://www.csun.edu/science/credential/cset/cset-physics/ppt/light\&color.ppt (13 August 2014).

[10] "light," Encyclopedia Britannica, http://www.britannica.com/EBchecked/topic/340440/light (15 August 2014).

[11] "Rainbow Formation," The Physics Classroom, http://www.physicsclassroom.com/class/refrn/u1414b.cfm (13 August 2013).

[12] "Science Activity: Secret Colors in a Black Marker!," Exploratorium, https://www.exploratorium.edu/science_explorer/black_magic.html (13 August 2014).

[13] "Ultimicron: The Ultimate LCD - Panel," EPSON News Release, http://global.epson.com/newsroom/2012/news_20120306_2.html (15 August 2014).

[14] "The 3 types of Projector explained: LCD, DLP, CRT," Oriphus, afterdawn.com, http://forums.afterdawn.com/threads/the-3-types-of-projector-explained-lcd-dlp-crt.291323/ (15 August 2014).

[15] “4-Color Process Printing vs. Spot Color Printing," The Leslie Company, http://leslieco.com/?p=15 (15 August 2014).

[16] "colour blindness," Encyclopedia Britannica, http://www.britannica.com/EBchecked/topic/126712/colourblindness (15 August 2014).

[17] "Can dogs see color - what color is their world?", Dog Blog World, http://www.dog-blog-world.com/can-dogssee-color/ (15 August 2014). 